\title{
EFFECTS OF CONTROLLED WHOLE-BODY VIBRATION TRAINING ON FUNCTIONAL PERFORMANCE AMONG HEALTHY OLDER ADULTS: A 6-WEEK PILOT STUDY
}

\author{
F. Saucedo ${ }^{1}$, E.A. Chavez ${ }^{2}$,H.R. Vanderhoof ${ }^{2}$, J.D. Eggleston ${ }^{2,3}$
}

\begin{abstract}
Background: Falling is the second leading cause of injury-related death worldwide and is a leading cause of injury among older adults. Whole-body vibration has been used to improve fall risk factors in older adults. No study has assessed if vibration benefits can be retained over time. Objectives: The aims of this study were to examine if six-weeks of whole-body vibration could improve fall risk factors and to assess if benefits associated with the training program could be sustained two months following the final training session. Design and Setting: Repeated measures randomized controlled design. Participants: Twenty-four independent living older adults were recruited and were randomly assigned to the WBV or control group. Intervention: Participants performed three sessions of whole-body vibration training per week with a vibration frequency of $20 \mathrm{~Hz}$ or with only an audio recording of the vibration noise. An assessment of fall risk factors was performed prior to, immediately following, and two-months after the completion of the training program. Main Outcome Measures: Fall risk factors including functional capacity, mobility, strength, and walking speed were assessed pre-training, post-training, and two-months post-training. Results: Seventeen participants completed the study. No improvements $(p<0.05)$ between groups were found in the measures of physical performance. Conclusions: Findings revealed that six weeks of whole-body vibration is not effective in improving fall risk factors or producing benefits post-training.
\end{abstract}

Key words: Vibration exercise, fall prevention, muscle strength, retention, functional mobility.

\section{Introduction}

Falling is the second leading cause of injury-related death worldwide (1) and is a leading cause of injury among older adults. More than one-third of older adults in the United States will fall in a given year (2) and considering the increased prevalence of falls among this age group (i.e. 60 years and older), this presents a significant global healthcare and economic issue (3). Reports have shown that up to 29 million community dwelling older adults experience falls each year, resulting in 7 million injuries requiring medical treatment (4).

Age-related changes in physical performance expose older adults to increased falls risk and high fall-related morbidity (5) and thus, the main focus of interventions should focus on improving performance in areas related to falls. Traditional approaches such as aerobic or resistance training have attempted to

\footnotetext{
1. Department of Kinesiology, Penn State Altoona, Altoona, PA, USA; 2. Interdisciplinary Health Sciences Doctoral Program, The University of Texas at El Paso, El Paso, TX, USA; 3. Department of Kinesiology, The University of Texas at El Paso, El Paso, TX, USA

Corresponding Author: Fabricio Saucedo, PhD, Department of Kinesiology, Penn State Altoona, 300 Ivyside Park, Rm. 203, Altoona, Pennsylvania 16601, USA, Tel: +1-814-949-5307, E-mail: fns5045@psu.edu

Received March 3, 2021
}

Accepted for publication May 6, 2021 improve physical performance to mitigate risk factors associated with increased falls, such as muscle weakness, decreased mobility sensory loss, and deficits in balance (6). However, despite significant efforts, limitations such as physical ability (7) have prevented access and have affected long-term adherence to traditional exercise programs.

Recently, whole-body vibration (WBV) has been utilized to train older adults to improve physical performance (8). Compared to traditional exercise programs, WBV is less strenuous, can be portable and cost-effective, and requires minimal exercise experience (8). Participants stand on the vibration platform and experience low frequency mechanical stimulation, which stimulates the muscle spindles (9). This activates alphamotor neurons in the central nervous system which elicits tonic muscle contractions in the lower extremities (9). The transmission of vibrations and oscillations to the human body can lead to physiological changes on numerous levels (10). Studies have demonstrated that vibration can nurture coordination and improve muscle strength, which can be an effective method in improving postural control in older adults (11). This has been demonstrated in studies examining six-week training periods, which have demonstrated neuromuscular adaptations and increases in neural activation (12), which in turn might 
have aid physical performance or lead to acute benefits.

Although several studies have examined the effects of WBV in older adults, only four other studies have assessed if WBV benefits can be retained over time (1316). These studies assessed performance after a washout period of three weeks, three months, and six months, respectively, and found that participants were not able to sustain WBV training benefits. Therefore, it is not certain if WBV exercise can produce health and performance benefits following the cessation of a training program. Thus, the purposes of this study were to examine if a six-week course of WBV training could improve fall risk factors and to examine if benefits of WBV could be retained over a two-month period after completing the program. It was hypothesized that six weeks of WBV would improve fall risk factors compared to a control $(\mathrm{CON})$ group. Additionally, it was hypothesized that benefits associated with WBV would be sustained over the two-month period following the completion of the WBV program.

\section{Methods}

\section{Participants}

Twenty-four older adults between the ages of 60-85 with physician clearance, no history of neurological, cognitive, musculoskeletal, cardiovascular, or known gait impairments were recruited for the study (Figure 1). Participants were recruited via advertisements on social media and flyers throughout the Greater El Paso, Texas Region and through our contacts with different institutes and hospitals in the city of El Paso. From the initial 24 recruits, only seventeen participants (13 female and 4 male) ages (70.4 \pm 6.2 years) completed the study (Table 1); seven participants did not complete the study due to research restrictions from the COVID-19 global pandemic. Participants were randomly assigned into one of two groups (WBV $n=9$ or $\mathrm{CON} n=8$ ) using a random number generator and were briefed on all procedures. Participants were not aware of their assignment into the CON or WBV group and this information was withheld for the duration of the study. Participants provided written informed consent approved by the University's Institutional Review Board. This was a pilot study utilizing a randomized controlled design and was performed in accordance with the ethical standards as described by the 1964 Declaration of Helsinki.

\section{Assessment of functional mobility}

Functional mobility was evaluated using the timedup-and-go test (TUG). Participants rose from an armed chair with no use of the arms, walked forward three meters, crossed a marked line on the floor and returned to the original seated position. The test began when the investigator said "go" and ended when the participant returned to the seated position. Participants were instructed to complete the task quickly and safely. The total time taken to complete the task at maximal speed was used for analysis.

Table 1

Group demographic parameters for participants in the whole-body vibration and control group

\begin{tabular}{lccc}
\hline \multicolumn{1}{c}{ Parameter } & WBV $(\mathbf{n}=9)$ & CON $(\mathbf{n}=8)$ & p value \\
\hline Age (years) & $71.4 \pm 7.1$ & $69.1 \pm 5.2$ & 0.730 \\
Sex (female) & 7 & 6 & 0.563 \\
Body height $(\mathrm{m})$ & $1.6 \pm 0.1$ & $1.6 \pm 0.1$ & 0.355 \\
Body mass $(\mathrm{kg})$ & $76.7 \pm 13.3$ & $82.9 \pm 18.7$ & 0.792 \\
\hline
\end{tabular}

Values are $n$, mean \pm standard deviation, or as otherwise indicated

Figure 1

Study flowchart outlining participant recruitment, randomization, and course of study

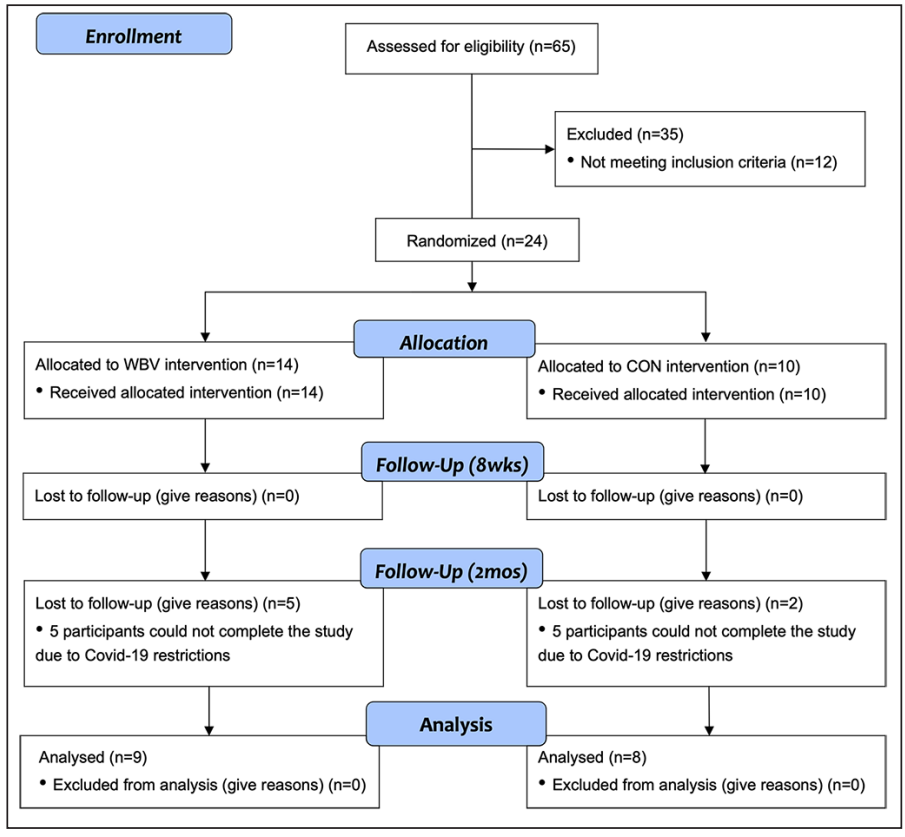

\section{Assessment of functional capacity}

Functional capacity was assessed using the two-minute walk test (2MWT). Participants were instructed to walk for two minutes between two cones set 30.48 meters apart. Participants were permitted to rest during the two-minute test but were made aware that the timer would continue to run until time expired. Total distance traveled during the two-minutes was used as a measure of functional capacity. 


\section{Assessment of walking speed}

Participants performed three walking trials of selfselected normal gait along a 10 -meter straight walkway (10MWT). Time to complete each trial in seconds was recorded to the hundredth second. The mean of the of the three trials was used for analysis.

\section{Assessment of muscle strength}

Maximum isometric torque of the quadriceps and hamstrings was assessed for all participants. Participants completed a standardized warm-up and test protocol on a motor-driven dynamometer (System 3, Biodex Medical Systems, Inc., Shirley, NY). The knee extension/flexion isometric strength assessment was performed bilaterally, in a seated position on a posterior-inclined $\left(15^{\circ}\right)$ chair. The proximal portion of the leg, pelvis, and shoulders were stabilized with safety belts. The rotational axis of the dynamometer was aligned with the mediolateral kneejoint axis and connected to the distal end of the tibia using an adjustable rigid lever arm. The three-dimensional positions of the rotational axis, the position of the chair, and the length of the lever arm were recorded and were identical for the strength assessment during the other testing sessions (e.g., post-training and twomonth follow-up). Each participant performed three repetitions, each lasting seven seconds for both flexion and extension on the dominant and non-dominant leg. Leg dominance was identified by asking the participant which leg would be used to kick a ball. One-minute resting periods were administered between repetitions. The average maximum torque normalized to body mass (Newton-meter/kilogram $(\mathrm{Nm} / \mathrm{kg})$ ) from the three trials was used for analysis.

\section{Training Intervention}

During each training, participants in the WBV group completed one set of vibration training. The training was intermittent with one-minute vibration sessions followed by a one-minute rest, for a total of 10 minutes (Figure 2 ). To avoid adverse effects or discomfort while on the vibration platform, knee flexion was maintained at $20^{\circ}$ (17). To minimize the shoe-dampening effect, participants stood on the platform barefoot. A side-alternating vibration platform (Galileo Med-L, Germany) was used and is depicted in Figure 2. The platform rotated about an anteroposterior axis, so positioning the feet farther from the axis of rotation would result in larger-amplitude vibration. The vibrator provided stimulation at fixed frequency of $20 \mathrm{~Hz}$ with the vibration amplitude set to 1.3 $\mathrm{mm}$, a setting designed to stimulate the stretch reflex and promote muscle function (Galileo Med-L, German). This vibration frequency was selected to maximize comfort and retention in the protocol and to reduce the risk of excess stimulation or resonating of the physiological systems (18). The assessments for physical performance described previously were performed in the morning hours prior to training (pre), immediately following the completing of the six-week WBV program (post), and two months after the completion of the protocol for retention (rtn). All sessions were performed in the laboratory at The University of Texas at El Paso all assessors were not blinded to participant group allocation.

\section{Figure 2}

Schematics of (a) the whole-body vibration timeline and protocol breakdown and (b) participant set-up on the side-alternation vibration platform. Vibrations were delivered intermittently at a frequency of $20 \mathrm{~Hz}$ and a vibration amplitude of $1.3 \mathrm{~mm}$

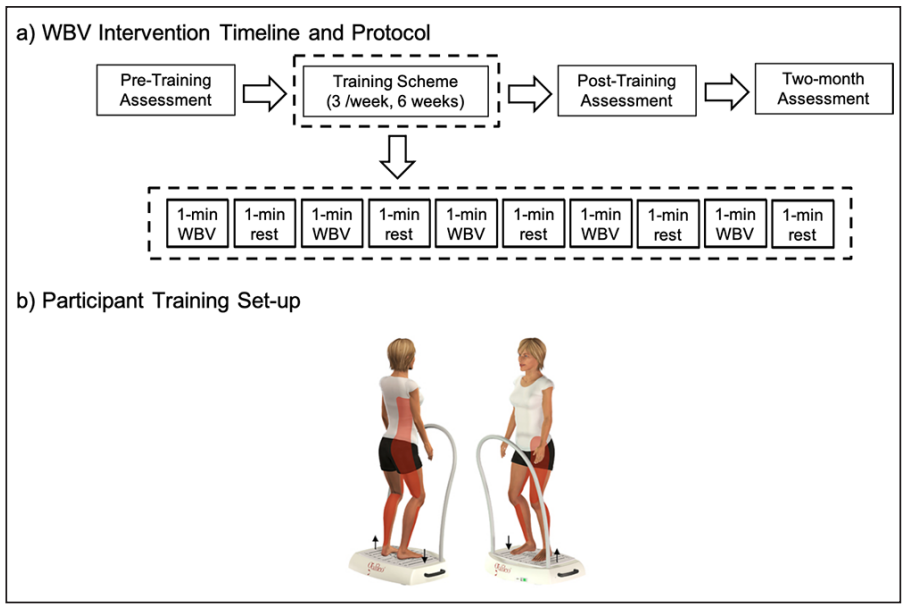

The CON group completed an identical program with no vibration. An audio recording of the vibrator motor was played during the session to mimic the sound of the WBV protocol (19).

Training sessions occurred three times per week, for six weeks. At least 24 hours were observed between consecutive training sessions. Successful completion of the programs occurred when each participant completed 18 sessions. Training sessions were supervised and conducted individually to monitor participant status and note any adverse mild effects potentially associated with training (itching, edema of the legs, soreness) (20). Participants were instructed to hold a stability bar attached to the vibration platform to minimize any fall risk.

\section{Statistical analyses}

An a priori sample estimate of 32 participants was calculated in G-Power 3.1 with a critical alpha-level set at 0.05 , a large effect size $(\mathrm{d}=1.03)$, and power of 0.80 . Analyses were performed using SPSS software version 24 (IBM, Armonk, New York). A Chi-Square Test was conducted to assess between group differences in baseline characteristics and Fisher's Exact Test was used to denote 
Table 2

Performance outcomes for the control and whole-body vibration group for all testing periods

\begin{tabular}{lccccccc}
\hline \multicolumn{1}{c}{ Variable } & \multicolumn{3}{c}{ WBV group $(\mathbf{n}=9)$} & \multicolumn{3}{c}{ CON group (n=8) } & p-value \\
& Pre & Post & Rtn & Pre & Post & Rtn \\
\hline Knee Strength (Nm/kg) & & & & & & & \\
Knee extensor (R) & $90.74 \pm 20.94$ & $87.23 \pm 22.36$ & $84.53 \pm 24.96$ & $98.80 \pm 30.04$ & $90.75 \pm 31.76$ & $93.51 \pm 22.87$ & 0.851 \\
Knee flexor (R) & $65.70 \pm 24.72$ & $65.77 \pm 15.35$ & $65.62 \pm 14.35$ & $66.56 \pm 21.62$ & $64.43 \pm 20.74$ & $65.46 \pm 18.48$ & 0.939 \\
Knee extensor (L) & $84.78 \pm 19.09$ & $92.78 \pm 17.97$ & $86.58 \pm 17.69$ & $103.49 \pm 26.19$ & $101.70 \pm 28.76$ & $96.51 \pm 16.99$ & 0.090 \\
Knee flexor (L) & $56.64 \pm 22.04$ & $67.82 \pm 8.99$ & $69.33 \pm 11.92$ & $58.66 \pm 19.74$ & $63.15 \pm 16.73$ & $55.74 \pm 16.49$ & 0.142 \\
TUG (s) & $7.39 \pm 1.39$ & $7.07 \pm 0.96$ & $7.20 \pm 1.69$ & $7.07 \pm 1.30$ & $7.36 \pm 0.90$ & $7.16 \pm 1.36$ & 0.176 \\
10MWT (s) & $8.13 \pm 1.53$ & $7.79 \pm 1.44$ & $7.69+1.83$ & $7.85 \pm 1.21$ & $8.11 \pm 1.10$ & $6.78 \pm 1.33$ & 0.114 \\
2MWT (m) & $167.63 \pm 17.66$ & $177.08 \pm 20.46$ & $181.90 \pm 19.04$ & $168.62 \pm 23.80$ & $177.42 \pm 17.87$ & $173.13 \pm 27.90$ & 0.276 \\
\hline
\end{tabular}

TUG: Timed Up and Go test; 2MWT: Two-minute walking test; 10MWT: 10-meter walking test. p-value reflects between-group differences

significance. Repeated measures analysis of variance (ANOVA) was used to identify the effect of WBV training on muscle strength, and performance on the TUG, 2MWT, and 10MWT. The within subject factor was the time instances (pre vs. post vs. rtn) while group (WBV vs. $\mathrm{CON}$ ) served as the between subject factor. An alpha level of $p<0.05$ was used to determine statistical significance.

\section{Results}

Baseline characteristics are presented in Table 1. The Chi-Square Test revealed no differences in gender between groups and no differences were identified between groups in age (yrs.), height $(\mathrm{m})$, or mass $(\mathrm{kg})$. No significant time by group 2-way interaction was detected for any of the variables (Table 2), however, isometric extension of the left leg approached significance $(\mathrm{p}=0.090)$ (Figure. $4 \mathrm{~b})$. The ANOVA revealed a significant main effect of time for the 10MWT $(p=0.033)$ (Figure. 3a) and the 2MWT $(\mathrm{p}=0.013)$ (Figure. $3 \mathrm{~b})$, but not for the TUG test (Figure. 3c) or the right and left measures of leg strength (Figure. $4 a-b$ ). Mean and standard error values are displayed in Figures 3 and 4.

\section{Discussion}

The aims of this study were to examine if six-weeks of WBV training could improve fall risk factors in older adults and to examine whether WBV benefits could be retained at least two months after completion of the WBV program. It was hypothesized that participants in the WBV group would improve in all fall risk factors. Additionally, it was hypothesized that all performance benefits associated with WBV would be retained in participants after two months. Based on the study findings, the hypotheses were not supported.
Figure 3

Group means and standard error bars for (a)10MWT, (b) 2MWT, and (c) TUG Test for the pre-test (Pre), post-test (Post), and two-month retention (Rtn). Asterisk ( $\left.{ }^{*}\right)$ indicates within group differences $p<0.05$ for the duration of the study

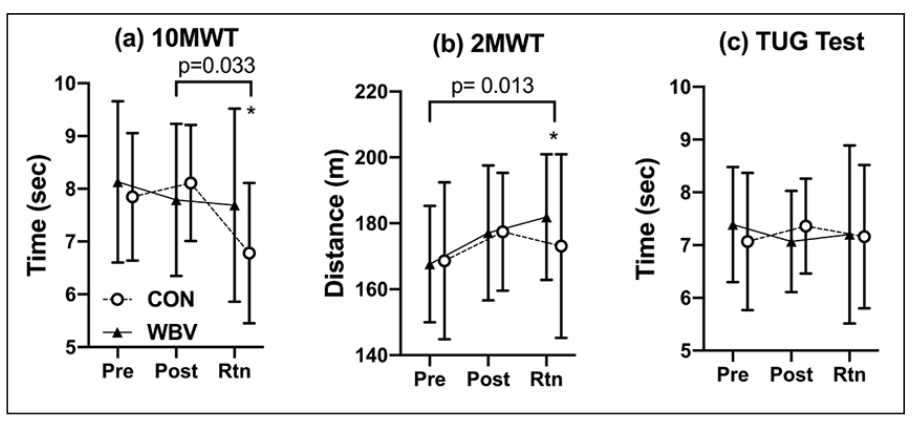

Other studies have also shown that WBV is no more effective than placebo conditions or traditional methods of intervention $(21,22)$. The findings from these studies as well as our study contrast those reported previously (23, 24). The study by Kawanabe and colleagues (2007) found that incorporating WBV training into a conventional regimen consisting of lower extremity strength exercises significantly improved walking speed in the 10MWT compared to the exercise-only group. Simão et al., (2012) determined that WBV signifcanlty improved distance walked during the 6MWT (similar to our 2MWT), and walking speed in the 10MWT. However, much like the study by Kawanabe et al., (2007), the participants in the study underwent a combination of WBV and squat therapy (24). Several other studies have reported performance improvements associated with WBV, but these too have combined exercise with a WBV regimen $(19,25)$. 
Figure 4

Group means and standard error bars for the (a-b) right and left max extensor torque and (c-d) right and left max flexor torque for the pre-test (Pre), post-test (Post), and two-month retention (Rtn)
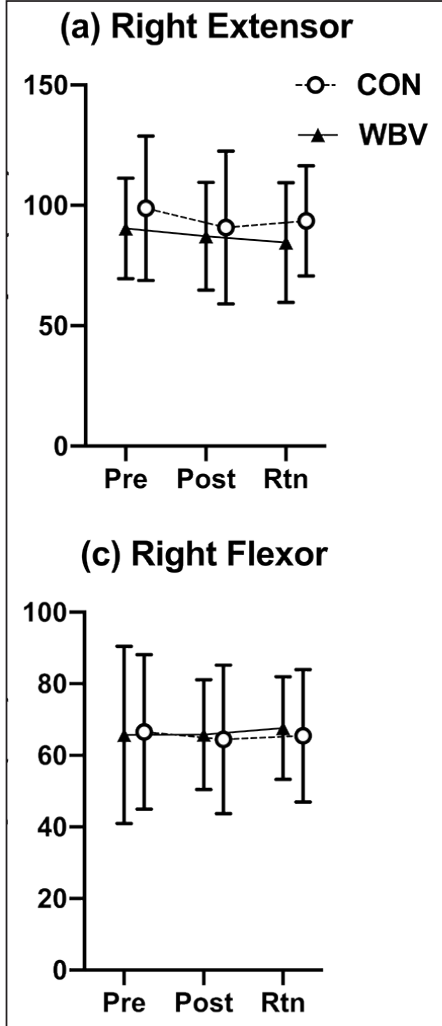

(c) Right Flexor (b) Left Extensor

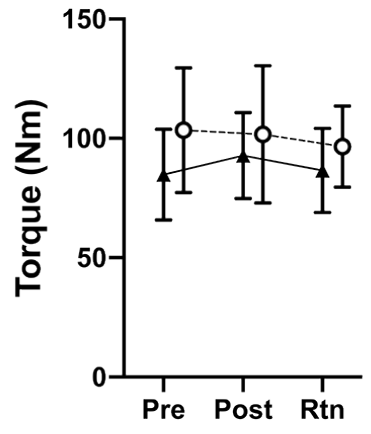

(d) Left Flexor

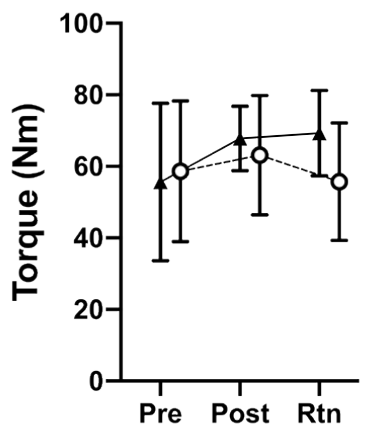

Few studies conducted previously have implemented protocols similar to our study. One study reported significant between group differences with participants in the WBV group showing greater increases in muscle strength and muscle hypertrophy compared to the control group (26). Another study reported significant improvements in participants who underwent 8-weeks of WBV. Participants experienced improvements in isometric knee extensor and flexor strength (8). This study did not include a control group, as our study, limiting their ability directly link performance benefits to the intervention.

Our study did not provide any evidence indicating any performance improvements linked to WBV. One possible reason for this outcome may relate to the duration of the study intervention. Other studies have commonly implemented WBV training periods lasting between three and eight months $(25,27)$. While the six-week period that we chose may be sufficient to yield neuromuscular adaptations or increase neural activation (12), which in turn might have aided physical performance or lead to acute benefits, it may be possible that six weeks does not suffice to obtain benefits from WBV. Other studies have implemented six-week WBV interventions in older adults and have reported improved balance and mobility/walking scores $(19,28)$. With the exception of the findings reported by Sitja-Rabert et al., (22), which found no improvements in physical performance in older adults after a six-week vibration intervention, studies implementing six-week interventions have shown improvements in fall risk factors and therefore other explanations for the findings in our study must be considered.

We acknowledge several limitations in this study. One possible limitation is that the training intensity and frequency were not adequate to elicit physiological changes linked to the improvements in the fall risk factors. The vibration frequency selected for the current study was $20 \mathrm{~Hz}$ and it was delivered intermittently for 60 -seconds for a total of five-minutes, three times weekly. Vibration frequencies ranging from 12.5 to 20 $\mathrm{Hz}$ have typically been classified as low-intensity, while frequencies from $30-50 \mathrm{~Hz}$ have been classified as highintensity (29). In theory, higher vibration frequencies elicit greater responses from the proprioceptors of the lowerextremities, however, many studies have shown that WBV interventions utilizing $20 \mathrm{~Hz}$ still result in improved performance outcomes (i.e. lower falls risk) (8). Another limitation that might have resulted in lack of significant findings in the present study is potentially attributed to the small sample size. Based on the a priori sample size estimation, a total of 32 participants was required to achieve sufficient statistical power. A posteriori poweranalysis revealed that with the 17 total participants that were recruited, the present study only yielded a statistical power of 0.22 at the 0.05 alpha level, thereby increasing the likelihood of type 2 error. Considering the smaller sample size composed of generally healthy and high-functioning participants, a ceiling effect could have resulted. The COVID-19 global pandemic impacted the study sample size, but future studies will aim to increase the study sample size to increase study power. One final limitation may be linked to the methodology that was utilized in the study. Isokinetic dynamometry was used to assess leg strength, specifically knee flexion and extension torque and no significant findings were found. While WBV can be effective in stimulating proprioception of the lower extremity, the vibratory stimulation is mainly targeted distally at the ankle joint because this is where the majority of the signal is dampened. Therefore, it would be more appropriate for future studies to examine ankle plantarflexion and dorsiflexion. This may also be beneficial as the plantarflexion and dorsiflexion play a vital role in the ankle mechanism for postural control.

The overall conclusion from this study was that sixweeks of WBV was not effective in improving physical performance on fall risk assessments among healthy older adults. While the findings from this study did not reveal statistically significant findings, there is one key strength which should be emphasized. This study represents the only one of a few studies to have looked at possible retention of benefits in older adults. Although not significant, this study could potentially be utilized 
in the scientific community to modify and design future protocols to examine the effects of WBV on risk factors associated with falls and retention among older adults. Future studies are required to identify the full benefits of WBV on improving performance fall risk factors in older adults.

\section{Conflict of interest: None declared.}

Acknowledgments: The authors thank Bianca Tovar, Alyssa Olivas, Pearl Quintero, and Christian Sanchez for their assistance

Funding sources: This study was funded The University of Texas at El Paso Dodson Research Grant, the Texas American College of Sports Medicine Student Research Development Award, and a generous contribution from the Virtual Reality and Motor Control Research Laboratory directed by Dr. Jason Boyle.

\section{References}

1. World Health Organization, "Falls." https:// www.who.int/ news-room/ factsheets / detail/ falls (accessed Oct. 29, 2020).

2. W. P. Berg, H. M. Alessio, E. M. Mills, and C. Tong, "Correlates of Recurrent Falling in Independent Community-Dwelling Older Adults," J. Mot. Behav. 1997; 29 (1): 5-16.

3. M. E. Tinetti, "Preventing falls in elderly persons," N. Engl. J. Med. 2003; 348 (1): $42-49$.

4. A. Dellinger, "Older Adult Falls: Effective Approaches to Prevention," Current Trauma Reports. 2017; 3 (2): 118-123.

5. C. S. Florence, G. Bergen, A. Atherly, E. Burns, J. Stevens, and C. Drake, "Medical Costs of Fatal and Nonfatal Falls in Older Adults," J. Am. Geriatr. Soc. 2018; 66 (4): 693-698.

6. J. Woo, A. Hong, E. Lau, and H. Lynn, "A randomised controlled trial of Tai Chi and resistance exercise on bone health, muscle strength and balance in community-living elderly people," Age Ageing. 2007; 36 (3): 262-268.

7. J. H. Rimmer, "Barriers associated with exercise and community access for individuals with stroke," J. Rehabil. Res. Dev. 2008; 45 (2): 315-322.

8. F. Yang, G. A. King, L. Dillon, and X. Su, "Controlled whole-body vibration training reduces risk of falls among community-dwelling older adults," J. Biomech. 2015; 48 (12): 3206-3212.

9. A. Bogaerts, C. Delecluse, A. L. Claessens, W. Coudyzer, S. Boonen, and S. M. P. Verschueren, "Impact of whole-body vibration training versus fitness training on muscle strength and muscle mass in older men: A 1-year randomized controlled trial," Journals Gerontol. - Ser. A Biol. Sci. Med. Sci. 2007; 62 (6): 630-635.

10. K. H. Madou and J. B. Cronin, "The effects of whole body vibration on physical and physiological capability in special populations.," Hong Kong Physiother. J. 2008; 26: 24-38.

11. A. Bogaerts, S. Verschueren, C. Delecluse, A. L. Claessens, and S. Boonen "Effects of whole body vibration training on postural control in older individuals: A 1 year randomized controlled trial," Gait Posture. 2007; 26 (2): 309-316.

12. B. M. Baroni, R. Rodrigues, R. A. Franke, J. M. Geremia, D. E. Rassier, and M. A. Vaz, "Time course of neuromuscular adaptations to knee extensor eccentric training," Int. J. Sports Med. 2013; 34 (10): 904-911.

13. P. J. Marín et al., "Effects of vibration training and detraining on balance and muscle strength in older adults," J. Sport. Sci. Med. 2011; 10 (3): 559-564.

14. S. Y. Tseng, C. L. Lai, K. L. Chang, P. S. Hsu, M. C. Lee, and C. H. Wang, "Influence of whole-body vibration training without visual feedback on balance and lower-extremity muscle strength of the elderly," Med. (United States). 2016; 95 (5)
15. R. D. Pollock, F. C. Martin, and D. J. Newham, "Whole-body vibration in addition to strength and balance exercise for falls-related functional mobility of frail older adults: A single-blind randomized controlled trial," Clin. Rehabil. 2012; 26 (10): 915-923.

16. J. M. Crielaard et al., "Evaluation of the impact of 6-month training by whole body vibration on the risk of falls among nursing home residents, observed over a 12-month period: a single blind, randomized controlled trial," Aging Clin. Exp. Res.. 2014; 26 (4): 369-376.

17. M. Mikhael, R. Orr, F. Amsen, D. Greene, and M. A. F. Singh, "Effect of standing posture during whole body vibration training on muscle morphology and function in older adults: A randomised controlled trial," BMC Geriatr. 2010; 10 (1): 74.

18. C. Rubin, M. Pope, J. C. Fritton, M. Magnusson, T. Hansson, and K. McLeod, "Transmissibility of 15-Hertz to 35-Hertz Vibrations to the Human Hip and Lumbar Spine: Determining the Physiologic Feasibility of Delivering LowLevel Anabolic Mechanical Stimuli to Skeletal Regions at Greatest Risk of Fracture because of Osteoporosis," Spine. 2003; 28 (23): 2621-2627.

19. I. Bautmans, E. Van Hees, J. C. Lemper, and T. Mets, "The feasibility of whole body vibration in institutionalised elderly persons and its influence on muscle performance, balance and mobility: A randomised controlled trial [ISRCTN62535013]," BMC Geriatr. 2005; 5 (1): 17.

20. F. M. H. Lam, R. W. K. Lau, R. C. K. Chung, and M. Y. C. Pang, "The effect of whole body vibration on balance, mobility and falls in older adults: A systematic review and meta-analysis," Maturitas. 2012; 72 (3): 206-213.

21. E. Rocha-Vieira et al., "The Effect of Adding Whole-Body Vibration to Squat Training on the Functional Performance and Self-Report of Disease Status in Elderly Patients with Knee Osteoarthritis: A Randomized, Controlled Clinical Study," J. Altern. Complement. Med. 2011; 17 (12): 1149-1155.

22. M. Sitjà-Rabert, M. J. Martínez-Zapata, A. F. Vanmeerhaeghe, F. R. Abella D. Romero-Rodríguez, and X. Bonfill, "Effects of a Whole body vibration (WBV) exercise intervention for institutionalized older people: A randomized, multicentre, parallel, clinical trial," J. Am. Med. Dir. Assoc. 2015; 16 (2): 125131.

23. K. Kawanabe, A. Kawashima, I. Sashimoto, T. Takeda, Y. Sato, and J. Iwamoto, "Effect of whole-body vibration exercise and muscle strengthening balance, and walking exercises on walking ability in the elderly," Keio J. Med. 2007; 56 (1): 28-33.

24. A. P. Simão et al., "Functional performance and inflammatory cytokines after squat exercises and whole-body vibration in elderly individuals with knee osteoarthritis," Arch. Phys. Med. Rehabil. 2012; 93 (10): 1692-1700.

25. A. Bogaerts, C. Delecluse, S. Boonen, A. L. Claessens, K. Milisen, and S. M. P. Verschueren, "Changes in balance, functional performance and fall risk following whole body vibration training and vitamin D supplementation in institutionalized elderly women. A 6 month randomized controlled trial," Gait Posture. 2011; 33 (3): 466-472.

26. A. Machado, D. García-López, J. González-Gallego, and N. Garatachea "Whole-body vibration training increases muscle strength and mass in older women: A randomized-controlled trial," Scand. J. Med. Sci. Sport. 2010; 20 (2): 200-207.

27. K. S. Leung, C. Y. Li, Y. K. Tse, T. K. Choy, P. . Leung, and W. H. Cheung "Effects of 18-month low-magnitude high-frequency vibration on fall rate and fracture risks in 710 community elderly - A cluster-randomized controlled trial," Osteoporos. Int. 2014; 25 (6): 1785-1795.

28. O. Bruyere et al., "Controlled whole body vibration to decrease fall risk and improve health-related quality of life of nursing home residents," Arch. Phys. Med. Rehabil. 2005; 86 (2): 303-307.

29. J. Muir, D. P. Kiel, and C. T. Rubin, "Vibration Exercise Devices To Standing Adults," Sport Med. Aust. 2013; 16 (6): 526-531. 